\title{
Ring polymers in solution : topological effects
}

\section{J. des Cloizeaux}

Service de Physique Théorique, CEN-Saclay, Boîte Postale no 2, 91190 Gif sur Yvette, France

(Reçu le $1^{\text {er }}$ juin 1981, accepté le 19 août 1981)

\begin{abstract}
Résumé. - On étudie les effets de contraintes topologiques sur les propriétés d'anneaux polymères en solution. Quand les anneaux sont courts et rigides, la nature de ces effets peut être aisément comprise et un résultat simple est donné ici. Quand les anneaux sont longs et flexibles, la situation est complexe et une analyse plus subtile est nécessaire. Heureusement des études mathématiques récentes concernant le nombre d'enlacements de deux courbes conduisent à un résultat significatif. Cette information permet de développer des arguments montrant que les contraintes topologiques produisent essentiellement un accroissement de l'interaction locale de volume exclu; cet effet topologique pourrait donc être pris en compte dans le cadre des théories actuelles.
\end{abstract}

\begin{abstract}
The effect of topological constraints on the properties of ring polymers in solution are studied. When the rings are short and rigid, the effects can easily be understood and a simple result is given here. When the rings are long and flexible, the situation is complex and a more subtle analysis is needed. Fortunately recent mathematical studies concerning the linking numbers of two curves lead to a significant result. This information is used to argue that the topological constraints produce essentially an increase of the local excluded volume interaction; this topological effect could therefore be taken into account within the framework of current theories.
\end{abstract}

1. Introduction. - - The static properties of linear polymers in good solvents depend on excluded volume interactions and are now rather well known. The properties of ring polymers in good solvents depend not only on the same kind of excluded volume interactions but also on topological constraints. In spite of a few studies $[1,2]$ by computer simulation, the effects of these constraints remain almost unknown [3]. We expect now that good samples of long ring polymers will soon be available [4]. Thus, it seems worthwhile to reexamine this interesting but difficult question.

It is clear that short rigid rings and long flexible rings have very different properties; therefore we shall study successively these two limiting cases, the second one appearing especially interesting.

2. Short rigid rings in solutions. -- Short rigid rings in solutions have nearly a circular shape and can be considered in a first approximation as simple molecules. Their properties come from the fact that the rings cannot penetrate each other. The size of a ring can be defined by the radius of gyration $R_{\mathrm{G}}$ and the mean volume occupied by a polymer ring we define to be

$$
V_{\mathrm{G}}=\frac{4 \pi}{3} R_{\mathrm{G}}^{3}
$$

The effect of the constraints can be observed by measuring the osmotic pressure $\Pi$. This quantity can expressed in terms of the dimensionless parameter $\mathbb{C} V_{G}$ where $\mathbb{C}$ is the number of polymers per unit volume and the coefficients of the expansion of $\Pi$ with respect to $\mathbb{C} V_{\mathrm{G}}$ are pure numbers.

$$
\Pi \beta=\mathbb{C}\left[1+\frac{1}{2} G \mathbb{C} V_{\mathrm{G}}+\cdots\right]
$$

Here

$$
G=V / V_{\mathrm{G}}
$$

where $V$ is the excluded volume.

For circles of radius $R$, we found

$$
R_{\mathrm{G}}=R \quad V_{\mathrm{G}}=4 \pi R^{3} / 3 \quad V=8 \pi R^{3} / 3
$$

and therefore

$$
G=2 \text {. }
$$

For comparison, we remark that a similar calculation for non intersecting discs of radius $R$ gave us

$$
\begin{gathered}
R_{\mathrm{G}}=2^{-1 / 2} R \quad V_{\mathrm{G}}=2^{1 / 2} \frac{\pi}{3} R^{3} \\
V=\left(\frac{\pi}{2}+\frac{4}{3}\right) \pi R^{3}
\end{gathered}
$$

and therefore

$$
G=2^{-3 / 2}(3 \pi+8)=6.16
$$


For solid hard balls of radius $R$, we would get

$$
\begin{gathered}
R_{\mathrm{G}}=(3 / 5)^{1 / 2} R \quad V_{\mathrm{G}}=\frac{4 \pi}{3}(3 / 5)^{3 / 2} R^{3} \\
V=\frac{4 \pi}{3}(2 R)^{3}
\end{gathered}
$$

and

$$
G=8(5 / 3)^{3 / 2}=17.21 .
$$

Further terms of the expansion of the osmotic pressure could also be calculated but the calculation would be more complicated. In particular, it would be interesting to consider the case where $\mathbb{C} R^{d}$ is large. Then the rings would form layers of rings parallel to one another (nematic configuration).

It would also be possible to consider the case of slightly flexible rings and to calculate by perturbation the effects of this small flexibility.

All these calculations may be complicated but the basic ideas are simple. Thus, we feel that we understand and can control this limiting case rather well.

3. Long flexible rings. - - It is much more difficult to study the effect of topological constraints on the properties of long flexible ring polymers in a solvent. For simplicity we shall assume that the long flexible rings have the same topology as a circle (trivial knot). In this way, the problem is well defined. We must exclude all configurations in which two (or several) ring polymers are linked (i.e. knotted configurations); this means that a configuration is excluded when the rings cannot be separated from one another by continuous deformation without crossing. Unfortunately, there are no simple classifications of knots and no simple ways of determining whether or not two rings make a knot.

However, some information can be obtained by studying the linking number of two rings : $\mathfrak{C}_{\alpha}$ and $\mathrm{C}_{\beta}$. This simple topological invariant is the integer given by Gauss' formula [5] :

$$
I_{\alpha \beta}=\frac{1}{4 \pi} \int_{\mathrm{e}_{\alpha}} \int_{\mathrm{e}_{\beta}} \frac{\left(\mathrm{dr}_{\alpha} \wedge \mathrm{d} \mathbf{r}_{\beta}\right) \cdot \mathbf{r}_{\alpha \beta}}{r_{\alpha \beta}^{3}}
$$

If $\mathcal{C}_{\alpha}$ and $\mathcal{C}_{\beta}$ are not linked, the linking number is zero; consequently if the linking number of $\mathrm{C}_{\alpha}$ and $\mathrm{C}_{\beta}$ is not zero, $\mathrm{C}_{\alpha}$ and $\mathrm{C}_{\beta}$ are linked.

The reverse is less conclusive; in general, if the linking number is zero, $\mathcal{C}_{\alpha}$ and $\mathcal{C}_{\beta}$ may be linked or not linked; however they are not linked if $\mathcal{C}_{\alpha}$ and $\mathfrak{C}_{\beta}$ are plane convex curves.

Thus the problem could be simplified by making the approximate assumptions that the excluded configurations are those for which the linking number is not zero. Thus it is useful to obtain information concerning the linking numbers of random flexible rings $\mathrm{C}_{\alpha}$ and $\mathrm{C}_{\beta}$.

Let $\rho$ be the vector joining the centre of gravity of $\mathrm{C}_{\alpha}$ to the centre of gravity of $\mathrm{C}_{\beta}$. Let us fix $\rho$ and let us assume that the orientations and the shapes of $\mathrm{C}_{\alpha}$ and $\mathrm{C}_{\beta}$ are at random. By averaging over these orientations and shapes, we define the mean square linking number

$$
J(\rho)=\left\langle\left[I_{\alpha \beta}(\rho)\right]^{2}\right\rangle
$$

and the volume

$$
J=\int \mathrm{d}^{3} \rho J(\rho) .
$$

Incidentally, we note that, if $\mathrm{C}_{\alpha}$ and $\mathrm{C}_{\beta}$ were plane convex curves, we would obtain

$$
J=V
$$

where $V$ is the average excluded volume of the curves, but in general $J$ and $V$ are different quantities.

It happens that $J$ can be expressed in a very simple way as was shown recently by W. Pohl [6] and also by J. des Cloizeaux and R. Ball [7] and by B. Duplantier [8]. Consider random curves $\mathcal{C}_{\alpha}$ and $\mathrm{C}_{\beta} . J$ is given by [7]

$$
J=\frac{1}{8 \Pi} \int_{0}^{\alpha} \mathrm{d} r \mathcal{A}_{\alpha}(r) \mathcal{A}_{\beta}(r)
$$

where $\mathcal{A}_{\alpha}(r)$ and $\mathcal{A}_{\beta}(r)$ are characteristic functions of $\mathrm{C}_{\alpha}$ and $\mathrm{C}_{\beta}$. The function $\mathcal{A}(r)$ associated with a random closed curve $\mathcal{C}$ is defined by [6]

$$
\begin{aligned}
\mathcal{A}(r) & =\frac{1}{r} \int_{\mathrm{e}} \int_{\mathrm{e}} \mathrm{d} \mathbf{r}_{1} \cdot \mathrm{d} \mathbf{r}_{2} \theta\left(r-r_{12}\right) \\
& =\frac{1}{r^{2}} \int_{\mathrm{e}} \int_{\mathrm{C}}\left(\mathrm{dr}_{1} \cdot \mathbf{r}_{12}\right)\left(\mathrm{dr}_{2} \cdot \mathbf{r}_{12}\right) \delta\left(r-r_{12}\right)
\end{aligned}
$$

where $r_{1}$ and $r_{2}$ define respectively the positions of points $M_{1}$ and $M_{2}$ on $\mathcal{C}(\theta(x)$ is the step function). The integrals are taken along $\mathrm{C}$.

The function $\mathcal{A}(r)$ for a long flexible ring of length $L$ can be approximated by an expression of the form

$$
\mathcal{A}(r)=L \varphi(r / \lambda)
$$

where $\lambda$ is a persistence length. Using (3.4), we verify that $\varphi(0)=2$ and that $\varphi(x)$ goes to zero when $x$ becomes large. Thus we may write

$$
J \simeq \frac{L_{\alpha} L_{\beta}}{8 \Pi} \int_{0}^{\infty} \mathrm{d} r \varphi^{2}(r / \lambda)
$$

and we claim that, in general, the integral is convergent. Thus we obtain

$$
J \simeq K L_{1} L_{2} \lambda
$$

which is valid for large values of $L_{\alpha} / \lambda$ and $L_{\beta} / \lambda$. Here $K$ is a constant which depends on the nature of the random curves.

The fact that the preceding integral (3.6) converges is of course crucial and can be shown in different ways. 
For instance, we calculated $K$ for a long gaussian ring. In this case, the persistence length $\lambda$ can be defined by setting

$$
\begin{aligned}
& \left\langle\frac{\mathrm{d} \mathbf{r}\left(l_{1}\right)}{\mathrm{d} l_{1}} \frac{\mathrm{dr}\left(l_{2}\right)}{\mathrm{d} l_{2}}\right\rangle=\exp \left[-\left|l_{1}-l_{2}\right| / \lambda\right] \\
& \frac{\left|l_{1}-l_{2}\right|}{L} \ll 1
\end{aligned}
$$

and we found

$$
\begin{aligned}
& K=2(3 \Pi)^{-3 / 2} \int_{0}^{1} \mathrm{~d} x \times \\
& \times \int_{0}^{1} \mathrm{~d} y[x-\ln x+y-\ln y-2]^{-1 / 2} \simeq 0.136 .
\end{aligned}
$$

On the other hand, it is not difficult to show that if the ring is in a good solvent $\varphi(x) \propto x^{1-1 / v}$; the integral (3.6) also converges in this case $(v \simeq 0.6)$.

The preceding formula (3.7) seems to indicate that the curves wind around each other locally when they are nearly in contact and that large loops do not occur frequently.

We now guess an asymptotic form for $J(\rho)$. Let $\mathbf{r}_{\alpha}\left(l^{\prime}\right)$ be the vector joining the centre of gravity of $\mathrm{C}_{\alpha}$ to a point $\mathrm{C}_{\alpha}$ (of coordinate $l^{\prime}$ ) and $\mathbf{r}_{\beta}\left(l^{\prime \prime}\right)$ the vector joining the centre of gravity of $\mathcal{C}_{\beta}$ to a point of $\mathrm{C}_{\beta}$ (of coordinate $l^{\prime \prime}$ ). We suppose that

$$
J(\boldsymbol{\rho})=K \lambda \int_{0}^{L_{\alpha}} \mathrm{d} l^{\prime} \int_{0}^{L_{\beta}} \mathrm{d} l^{\prime \prime} \delta\left[\mathbf{r}_{\alpha}\left(l^{\prime}\right)-\mathbf{r}_{\beta}\left(l^{\prime \prime}\right)-\boldsymbol{\rho}\right]
$$

which is compatible with (eq. 3.7); actually the fact that $J$ is proportional to $L_{1}$ and $L_{2}$ shows that (3.10) is reasonable.

On the other hand

$$
J(\rho)=\left\langle I_{\alpha \beta}^{2}(\boldsymbol{\rho})\right\rangle .
$$

I he curves $\mathrm{C}_{\alpha}$ and $\mathrm{C}_{\beta}$ may turn around each other in one way at one place, in another way at another place. Let $n$ be the total number of loops. Thus $I_{\alpha \beta}(\rho)$ can be considered as the sum of positive and negative variables

$$
I_{\alpha \beta}(\rho)=\sum_{j=1}^{n} x_{j}
$$

where $x_{j}= \pm 1$.

For large alues of $L_{\alpha} / \lambda$ and $L_{\beta} / \lambda$ these variables can be considered as independent. Thus we have

$$
\left\langle I_{\alpha \beta}^{2}(\rho)\right\rangle=\left\langle\sum_{j=1}^{n} x_{j}^{2}\right\rangle=\langle n\rangle
$$

and therefore

$$
\langle n\rangle=J(\rho) \text {. }
$$

By combining equations (3.10) and (3.11), we come to the conclusion that the total number $n$ of loops of $\mathcal{C}_{\alpha}$ around $\mathcal{C}_{\beta}$ is proportional to the number of contacts of these curves.

We can now generalize this idea. We may assume that two pieces of curve make knots when they come close to each other and that otherwise the probability of making knots is rather low and unimportant.

Thus, we are led to the following conjecture : long ring polymers are subjected to topological constraints but these constraints produce only excluded volume effects.

According to this conjecture, the critical indices of ring polymers in good solvents should be the same as these of linear polymers in good solvents. However, ring polymers should be more soluble than linear polymers and their Flory temperatures $(\theta$ point $)$ should not exactly coincide.

The quantity $b$ which defines the interaction in the two parameter model has a dimension [9]

$$
b=(\text { length })^{4-d} \quad(d=\text { space dimension })
$$

Thus for ring polymers in solution the part of the interaction which results from topological constraints should be proportional to $\lambda$.

We may write

$$
b \sim K \lambda .
$$

However to find more precise values of $b$ a specific model has to be chosen and elaborate calculations are required.

Finally, we note that the results presented in a recent letter by Brereton and Shah [10] are compatible with ours.

4. Conclusion. -- The main result of this discussion is the conjecture that for long flexible rings, the topological constraints and the excluded volume interactions have similar effects. This idea has now to be tested by conducting experiments on long ring polymers. We also have to perform more sophisticated calculations and to see whether the results obtained agree with the preceding simple conjecture.

Acknowledgments. - The author is indebted to R. Ball and B. Duplantier for valuable discussions and criticisms. 


\section{References}

[1] Vologodsky, A. V., Lukashin, A. V. and Kamenetsky, F., Zh. Eksp. Teor. Fiz. 67 (1974) 1875, Sov. Phys. JETP 40 (1975) 932.

[2] Des Cloizeaux, J. and Mehta, M. L., J. Physique 40 (1979) 665.

[3] See however : Edwards, S. F., Proc. Phys. Soc. 91 (1967) 513 ; J. Phys. A. Gen. Phys. 1 (1968) 15.

[4] Benort, H., Private Communication.

[5] Alexandroff, P. and Hopf, H., Topologie I (Springer Berlın) 1935.
[6] PoHL, W., To be published in International Symposium in honor of N. H. Kuiper. Utrecht 1980. Lecture Notes in Mathematics (Springer Verlag).

[7] Des Cloizeaux, J. and Ball, R., To be published in Communications in Mathematical Physics.

[8] DuplantieR, B., Submitted for publication to Communications in Mathematical Physics.

[9] Des Cloizeaux, J., J. Physique 42 (1981) 635.

[10] Brereton, M. G. and Shah, S., J. Phys. A. Math. Gen. 14 (1981) L-51. 